\title{
The Role of Recruitment Analytics and Metrics in Targeted Recruitment Post Pandemic
}

\author{
Dr. Thiyagarajan $\mathrm{R}^{1}$ \\ $\left\{\right.$ thiyagu@psgim.ac.in $\left.{ }^{1}\right\}$ \\ Assistant Professor (Sr. Gr.), PSG Institute of Management ${ }^{1}$, India.
}

\begin{abstract}
COVID has become the accelerator for the workplace transformations of lifetime. Hiring talented candidates is crucial job to a company's success. The recruitment of employees is a challenging task. Even during recession, it is tough to fill certain style of jobs, the recruiters should make it crucial to take decisions, along with where to target and what content to deliver and how to group the workers for recruitment process. The research paper focuses on targeted recruitment in the pandemic times and the role of recruitment analytics and metrics. Predictive analytics in recruitment helps to reduce the time and cost of recruitment and also locate competences quicker. Streamline the extensive candidate database from facts and get a head begin on filling the skill pools with recruitment information analytics. When there is greaterposition for analytics, for recruitment will become vital in measuring KPIs. The paper concludes by stressing the need and role of recruitment analytics and metrics in enhancing the process of targeted recruitment, as the future is going to be, even more tech savvy. In the current situation, there arises a great need to align all the recruitment activities from strategy formulation to identifying, acquiring, developing, engaging and retaining the best talent to match with the new norm of work.
\end{abstract}

Keywords: Predictive analytics, Targeted recruitment, crucial decisions, recruitment information analytics, recruitment metrics, Recruiting.

\section{Introduction}

Recruitment is the process of trying to locate and inspire ability candidates to apply for current expected activities openings. In simple phrases, Recruitment techniques try and create a pool ofprofessional and experienced humans. In brief, recruitment is about sourcing the proper human beings, in proper time, in the proper area and in the right fee forattracting and keeping the right people is the cornerstone of a corporation's achievement. The key for hit recruitment is to bolster employer's relationship with each the current and ability of workers. Rather than retaining brief understanding with people who ought to fill vacant role, the focal point should be to high maintain lengthy-time period courting with human beings.

Recruitment is the first process that a firmtakes with thecapable candidates. It is through the recruitment that many individuals arerecognised in a firm, and eventually decided whether they will desire to work for it. A well prepared andcontrolled recruiting attemptbrings about excessive pleasant candidates, whereas a haphazard attempt will bring about mediocre ones. The recruitment technique informs qualified individuals about employment possibilities thatcreate a nice image of the organisations that provide enough facts of the job to helpcandidates who make comparisons with their qualifications and pursuits to create 
enthusiasm among some of the best applicants in order that toapply for the vacancies.Recruitment lends itself an ability supply of aggressive gain to a firm. An effective approach to recruitment can assist an organization correctly to compete for confined human resources. The company must pick a recruiting method that producesexcellent pool of candidates quick and fee successfully. Theresult of a bad recruitment speaks about its role in the corporation. The failure that generates a good enough number of capable applicants can show high in several methods. Further, when recruitment fails to satisfy organizational wishes for skills, is a regular response is to raise entry - level pay scales. This can distort traditional wage and profit relationships in organisations, resulting in unavoidable results.

\subsection{Targeted Recruitment}

Targeted recruitment is an approach utilized in recruitment advertising to explain the manner of narrowing downadvertising efforts on a specific target market is based totally on differentiating elements like geography, skill units, or enjoy. The targeted recruitment is a process that pay unique interest to people with positive characteristics while selecting them to fill a job application or vacancy. The primary winning of targeted scrutinizing is to diversify the group of workers and to offer the agency a big range of opionions to be chosen.By crafting a recruitment strategy that targets healthcare professionals like complete-time or in step withdiem nurses, for instance, have got the opportunity to cultivate a fantastic employment logo photo inside the healthcare space. Crafting a powerful targeted recruitment plan can assist the enterprise hire for difficult-to-fill requisitions. Targeted recruitment solves this by taking "post and pray" mentality out of job marketing. Whileorganisation may be specific about the criteria capability candidates to do a qualified task, to know that the applicationscould be qualified, ideal candidates. Focusing on advertising efforts on generating fine guarantees that spent is targeted on delivering applicants who fitfocused standards. When organisations seek to a choosetalents, every greenback put in the direction of recruitment marketing becomes even more precious. Targeted recruitment permitsto manage price range extra effectively and decreaseprice-in keeping with-lease because organisations may better allocatespend, accordingly taking into consideration a higher distribution of candidates. Analyse current hiring strategies Provide real-time reporting of recruitment tasks personalise the hiring techniques for unique talent Provide proactive expertise control services Enhance the employer emblem and Improve the best of applicants.

\subsection{Recruitment Analytics}

Recruitment strategies is the mixture of sources and predictive analysis that offers realtime records to help the organisation recruit quicker. Powerful recruiting analytics allows to explore each factor of the enterprise, flip information into actionable insights, and make higher recruiting choices faster. Make the maximum of each penny spent with our recruitment analytics software program. Monitor the reputation of process beginning with what number of great hires are received from every supply, discover which sources have the highest turnover rate, and lots greater. Use recruitment analysis dashboard to research where the exceptional performers discoverthe job list so thatattention can be made on that supply and drop ones that are not working nicely. Make the recruitment process greater cost-effective the usage of actionable insights with recruitment analytics equipment.Every exceptional hire offersan opportunity to expand the community, build new relationships, and notice talent for the future. Predictive analytics in recruitment allows reduce value per rent and discover capabilities faster. Streamline large candidate database from information and get a head begin on filling 
that expertise pool with recruitment facts analytics. When there are greater open positions analytics for recruitment will become important in measuring KPIs.

\section{Literature review}

Jillian R. Yarbrough (2017), The world has changed into electronic based systems. The system helps HR people to narrow down their work. There are many types in hiring candidates. One such example is free market hiring which allows the candidate to evolve in the market. Another such type is targeted hiring strategies which use technology, E- Targeted hiring to select the necessary candidates for organization.

Mamta Mohapatra, Priyanka Sahu (2017), Recruitment is shifted towards a data driven hiring process which involves analysing data. This study shows us that analysing data is an important aspect for recruitment. The efficiency of the candidate is analysed through various methods. The HR department in a company focuses on effective recruitment process which helps the company to work in a long term. Recruitment analysis, optimization are major terms in this approach.

D. Volpone, Kecia M. Thomus, Paula Sinisterra, and Lindsay Johnson (2013), In journal "Targeted Recruiting", Identifying Future Employees by Sanbrina, says that Minority recruitment has end up increasingly more critical topic for each researchers and practitioners. To shed mild on this subject matter, the paper discusses targeted diversity recruitment. Specifically, the chapter evaluations the literature surrounding strategic recruitment efforts as it relates to focused recruitment efforts. Further, this chapter discusses how organizations ship diffused and regularly unintended indicators at some stage in the recruitment method that impact how candidates examine corporations during recruitment activities.

Sabrina D. Volpone, Kecia M. Thomas, Paula Sinisterra, and Lindsay Johnson (2013), Minority recruitment is done by targeted diversity recruitment, diversity recruitment done through diversity messages and images in the formal recruitment advertisements. Organizations send subtle and unintentional signals to the recruiters. Personnel recruitment, diversity recruitment, minority employees and signal theory are all important topics in Targeted recruitment.

Daniel A. Newman Julie S. Lyon (2009), they gave a note thatnoting the presumed tradeoff between variety and overall performance desires in contemporary selection practice, the authors complicated on recruiting-based techniques for avoiding destructive impact while retaining combination character productivity. For sensible recruiting impact sizes, this sort of recruiting strategy substantially will increase common overall performance of hires and percentage of hires from the underrepresented group. Further effects from a policy-shooting look at offer preliminary guidance on how functions of organizational photo can attract applicants with activity-associated personalities and talents, similarly, to attracting candidates based on demographic heritage in recruitment efforts.

James A. Breaugh (2009), Hiring the talented individuals is a critical process. In this recruiting is a challenging task that covers all the areas of recruiting who can be recruited, the apt timing of recruitment, the message to be sent for recruitment, the details of visit to the organisation, review and evaluation of recruitment activities. 


\section{Research Model}

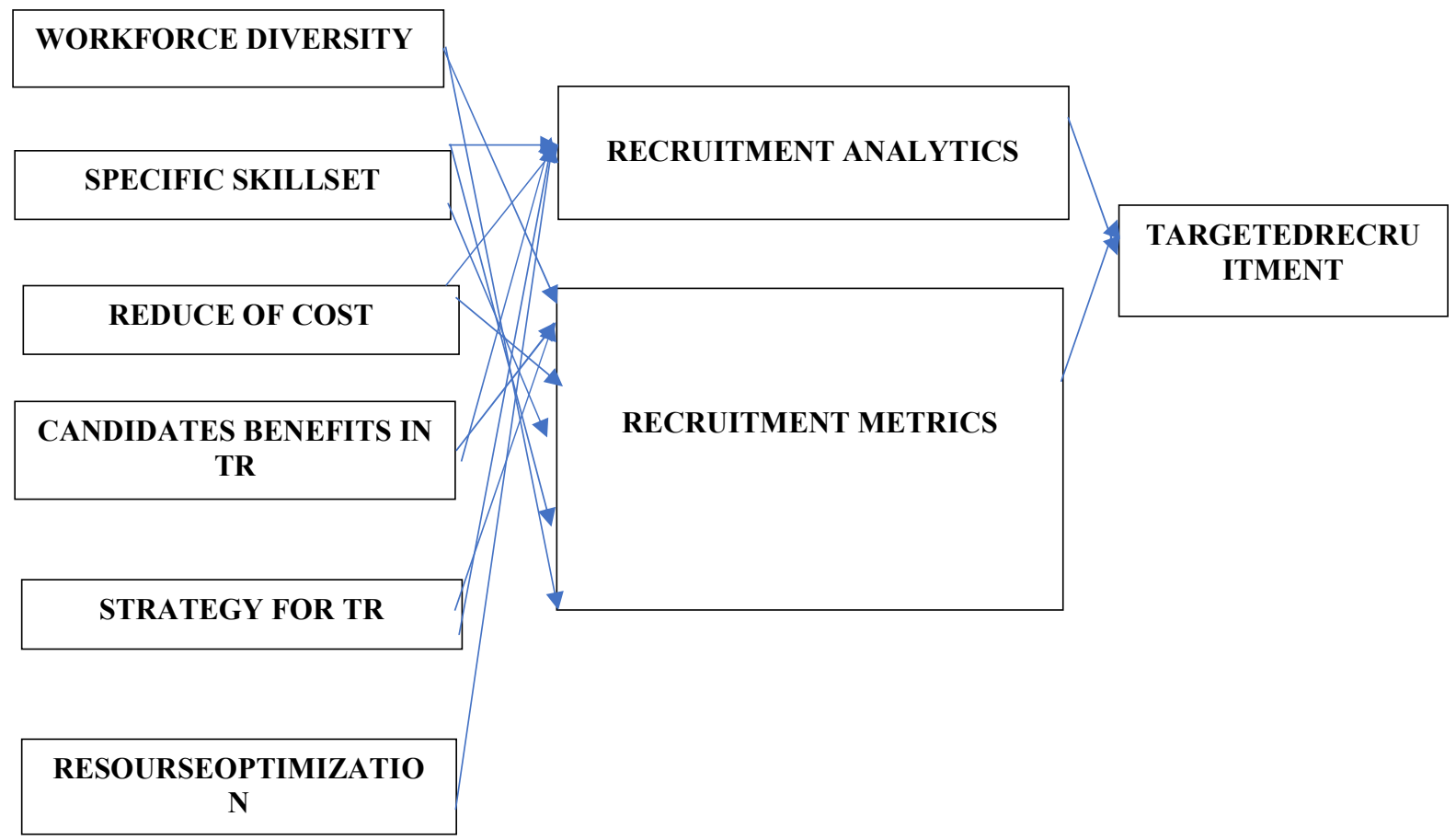

\section{Research Methodology}

The research is based on how recruitment analytics and recruitment metrics make an effect in targeted recruitment in organizations. The targeted recruitment facilitates the HR crew to sell balance within agency, exceptional hiring resources to hire and to decorate the sourcing of the first-rate candidates for one among the roles inside the corporation to help lessen fees and to better understand the elements that are influencing the hiring system. Recruiting talented individuals play an important rolein an organization's success, to hire the most talented the recruitment process must be strong which is difficult, the method and strategies in recruiting a talented individual for specific job functions must be unique as it is the critical importance to the HRM and organizations they represent.

The most method used by organizations is recruitment analytics and metrics, using the data driven metrics and insights improve the sourcing of the best candidates for different roles in an organization and helps in understanding the factors that influence the hiring process. The time to hire talented individuals for the organization is completely reduced by recruitment analytics and metrics using tools like online dashboards and HR analytics software during the hiring process. The data generated during the recruiting process is now in the form of 
recruiting metrics or key performance indicators which shows how effectively and efficiently the organization is recruiting, the process also includes clear understanding of organizations mission, forecasting, training managers on the targeted hiring process and creating a dynamic recruiting strategy. In this way recruitment analytics, metrics and KPIs can minimize long term experience and maximize recruiting talented individuals for the organization.

\section{Findings and Suggestions}

Every outstanding rent offers a possibility to expand community, construct newrelationships, and note abilities for the future. Predictive analytics in recruitment lets lessen fee constant with hire and find talents faster. Streamline large candidate database from statistics and get a head start on filling that skills pool with recruitment records analytics. When there are more open positions analytics for recruitment turns into essential in measuring KPIs.Make the most of each penny spend with our recruitment analytics software program. Monitor the fame of challenge beginning, track what number of exceptional hires have become from each supply, perceive which property have the satisfactory turnover rate, and plenty greater. Use recruitment assessment dashboard to research where first-rate performers decided activity listing so that organisations can consciousness on that source and drop ones that are not running nicely. Make recruitment method greater price-effective the usage of actionable insights with recruitment analytics tools. Recruitment metrics isan important thing to assess the overall performance of the recruitment process in an organisation. It is challenging to have measurable recruitment dreams and accurate records that tracks the key performance and tells where it stands, whenorganisations are trying to make game-converting hires.Wise use of recruiting metrics enables to evaluate facts from recruiting procedure towards company dreams for the sort of perception wants to hold and enhance recruiting practices.

\section{Conclusion}

Recruitment may be a manner that has evolved appreciably throughout the previous couple of decades. starting from a confined platform of setting method commercials in newspapers to current tendencies of social media enlisting and use of the many shrewd instrumentation for wonderful work analysis, this trade has developed to a multi-billion-dollar trade, shift from the economic age to facts age has created it important for all huge or small-scale agencies to accumulate applicable records helpful in choice creating at $\mathrm{C}$-Suite stage. Metrics like style of important vacancies empty past the prescribed rent time has direct result on business wishes and is applicable to the C-degree executives. Th paper highlights some regions wherever analytics could also be wont to assist build successful enlisting technique for the agency. when analysis of the corporation statistics, acting correlations of recruiting parameters with overall performance rankings, and drawing inferences from famed analysis and writings, it should be complete that the usage of analytics in enlisting will facilitate agencies take higher choices within the long run. There may be millions of ability venture within the operational potency of analytics in process and in loss of the adept personnel in 60 minutes domain WHO use analytics to derive the suggests that out of applicable information. however, businesses wish to require the initiative to 
accumulate records that's helpful for key creating method and use the required equipment's and measures to draw the inferences from the data. It has to be compelled to pay cash on schooling staff to figure on huge count of worker statistics to form inferences.

Human's timetable at companies includes bound vital business enterprise issues and also the wish Resources to peak to those challenges has ne'er been a lot of correct. to extend capability for depth analysis, the appliance of analytics may be a key token to form 60 minutes a strategic characteristic in any corporation throughout enlisting. However, 60 minutes should think about that analytics isn't continuously a 'one-and-executed' technique. To rent the standard talent, and may perpetually notice steps to develop the enlisting technique. choices while not a doubt helps to double the strength associate degreed remove weak spots within the enlisting method of an organisation to fulfil each would like of the corporate.

\section{References}

[1] Barber (2014). An Empirical Analysis of Recruitment and Selection Practices in the Public Sector, International Journal of Management Sciences and Business Research, 2014 ISSN (2226-8235) Vol-3, Issue 12.

[2] Emilio J. Castilla (2005). Social Networks and Employee Performance in a Call Centre

[3] AJS Volume 110 Number 5 (March 2005): 1243-83. 1243.

[4] Daniel A. Newman, Julie S. Lyon, (2009). Recruitment efforts to reduce adverse impact: Targeted recruiting for personality, cognitive ability, and diversity.Journal of Applied Psychology, 2009, Vol. 94, No. 2, $298-317$.

[5] Gamage, (2014). The impact of recruitment and selection criteria on organizational performance, Global Journal of Human Resource Management Vol.3, No.2, pp.22-33, March 2015.

[6] James A. Breaugh, (2009). Employee Recruitment,Recruiting and Attracting Talent, Vol.64:389416.

[7] Mamta Mohapatra, Priyanka Sahu., (2017).Optimizing the recruitment funnel in an ITES company: an analytics approach, Procedia computer science volume 122,2017, pages 706-714. Vol.64:389416.

[8] Sabrina D. Volpone, Kecia M. Thomas, Paula Sinisterra, and Lindsay Johnson (2013). Targeted recruiting: identifying future employees, The oxford handbook of recruitment, August 2013.

[9] Sandra Durcevic (2021) How Recruitment Metrics \& Dashboards Can Help Improve Hiring New Candidates, The datapine Blog, January 28th, 2021. 